\title{
Schnelle Regelung von linearen Systemen mit Stellgrößenbeschränkungen
}

\section{Fast Control of Linear Systems with Input Constraints}

Hendrik Lens und Jürgen Adamy

\begin{abstract}
In diesem Artikel wird eine nichtlineare Zustandsrückführung für Regelstrecken mit linearer Dynamik und einer Stellgrößenbeschränkung vorgestellt. Die Methode ermöglicht sehr schnelle Regelvorgänge. Erreicht wird dies durch die Verknüpfung sättigender und weicher strukturvariabler Regler. Es wird ein effizientes, LMI-basiertes Entwurfsverfahren zur Bestimmung der Reglerparameter vorgestellt. Bei Nachoptimierung der Parameter mittels Suchverfahren erreicht die Regelung eine Güte, die sehr nah an die zeitoptimale Lösung heranreicht.

This article presents a nonlinear control feedback for systems with linear dynamics and input constraints. The method allows very fast control action. The excellent performance is reached by combining saturation control with soft variable-structure controllers. An efficient LMI based design algorithm for the parameterization of the controller is presented. Optimizing these parameters using local nonconvex optimization provides a performance which is extremely close to the time optimal solution.
\end{abstract}

Schlagwörter: Weiche strukturvariable Regelung, fast zeitoptimale Regelung

Keywords: Soft variable-structure control, nearly time-optimal control

\section{Einleitung}

Jedes reale Regelsystem ist mit einer Stellgrößenbeschränkung behaftet. Im Bereich der linearen Regelungstheorie ist im Fall einer solchen Beschränkung sicherzustellen, dass die Regelung diese nicht verletzt, da sonst lineare Methoden zur Analyse des Systemverhaltens ihre Gültigkeit verlieren. Dies führt im Gegenzug dazu, dass die Regelung sehr viel langsamer wird als es eigentlich möglich wäre. Die Beschränkung der Stellgröße spielt demnach insbesondere dann eine große Rolle, wenn man einen möglichst schnellen Regelvorgang erreichen möchte.

Ein Ansatz, einen schnelleren Regelvorgang zu erreichen, besteht darin, zuzulassen, dass die kommandierte Stellgröße in die Sättigung geht. Dann muss die Sättigung allerdings in den Stabilitätsbetrachtungen berücksichtigt werden. Beispiele solcher Ansätze finden sich u.a. in [10;11].

Alternativ kann dazu übergegangen werden, mit Absicht einen nichtlinearen Regler einzusetzen. Die Nichtlinearität kann dann dazu genutzt werden, die Performance zu verbessern. Auch dazu existieren mehrere Ansätze, wie beispielsweise $[1 ; 3 ; 6 ; 8 ; 13 ; 14 ; 18]$.

Die in diesem Artikel vorgestellte Struktur greift mit weichen strukturvariablen Reglern mittels impliziter LjapunovFunktion [1;3] einen nichtlinearen Ansatz auf. Dieser Regler wird um die Möglichkeit erweitert, mit der Stellgröße in den gesättigten Bereich zu gehen. Durch diese Neuerung wird eine außerordentlich hohe Regelgeschwindigkeit erreicht, die bei richtiger Parameterwahl beinahe zeitoptimal sein kann. Eine Kombination zwischen einer weichen strukturvariablen Regelung und einer Sättigungsregelung, die $\alpha$-Regelung, wurde auch schon in [6] vorgeschlagen. In [9] und [12] werden ebenfalls Konzepte vorgestellt, die als sättigende weiche strukturvariable Regler bezeichnet werden können.

Betrachtet wird ein System mit der linearen Dynamik

$$
\begin{aligned}
& \dot{\mathbf{x}}=\mathbf{A} \mathbf{x}+\mathbf{b} u, \\
& y=\mathbf{c}^{\mathrm{T}} \mathbf{x},
\end{aligned}
$$


wobei $\mathbf{x} \in \mathbb{R}^{n}$ der Zustandsvektor, $u \in \mathbb{R}$ der Systemeingang und $y \in \mathbb{R}$ der Systemausgang ist. Der Eingang ist beschränkt durch

$$
|u| \leq u_{\max } .
$$

Die möglichen Anfangsbedingungen werden in der Menge $\mathcal{X}_{0}$ zusammengefasst:

$$
\mathbf{x}_{0}=\mathbf{x}\left(t_{0}\right) \in \mathcal{X}_{0} \subset \mathbb{R}^{n} .
$$

Außerdem wird davon ausgegangen, dass das Paar (A, b) vollständig steuerbar ist.

Die betrachtete Zielsetzung ist nun, eine Zustandsrückführung zu finden, welche den Zustandsvektor $\mathbf{x}$ des Systems (1) für alle möglichen Anfangszustände $\mathbf{x}_{0} \in \mathcal{X}_{0}$ so schnell wie möglich asymptotisch zum Ursprung führt.

Ein sehr einfacher Regler für das System (1) ist eine nichtsättigende lineare Zustandsrückführung

$$
u=-\mathbf{k}^{\mathrm{T}} \mathbf{x}, \quad\left|\mathbf{k}^{\mathrm{T}} \mathbf{x}(t)\right| \leq u_{\max } \forall t>t_{0}, \mathbf{x}_{0} \in \mathcal{X}_{0} .
$$

Der Vorteil dieses linearen Ansatzes besteht darin, dass die Analyse mit Methoden der linearen Regelungstheorie durchgeführt werden kann. Dieser Vorteil hat jedoch seinen Preis: Der Regler muss so gewählt werden, dass die Stellgröße $u$ für alle Trajektorien, die in $\mathcal{X}_{0}$ starten, innerhalb der sich aus der Stellgrößenbeschränkung ergebenden Begrenzung bleibt. Somit ist die erreichte Regelgüte deutlich schlechter, als sie sein könnte.

Es gibt verschiedene Möglichkeiten, dieses Problem zu umgehen und eine bessere Regelgüte $\mathrm{zu}$ erzielen. Zunächst ist es möglich zuzulassen, dass die lineare Rückführung in die Sättigung geht. In diesem Fall muss die Sättigung bei der Betrachtung der Stabilität mit berücksichtigt werden. Wie dies geschehen kann, ist bekannt [11] und wird in Abschnitt 2 kurz dargestellt.

Alternativ dazu kann man zu einer nichtlinearen Rückführung übergehen. Hierbei stellt sich die Frage, wie diese nichtlineare Rückführung gewählt werden soll, sodass eine gute Regelgüte erreicht und gleichzeitig die Stellgrößenbeschränkung eingehalten wird. Ein extremes Beispiel einer solchen Regelung ist die zeitoptimale Regelung. Diese weist jedoch gravierende Nachteile auf. Unter anderem existiert keine allgemein gültige Methode zur Bestimmung des Regelgesetzes. Außerdem wechselt die Stellgröße sprunghaft zwischen den Grenzen der Beschränkung $u_{\max }$ und $-u_{\max }$. Die Tatsache, dass zeitoptimale Regelungen immer die maximale Stellgröße beaufschlagen, legt nahe, dass ein Regler den Stellbereich möglichst gut ausnutzen sollte, um eine schnelle Regelung zu erzielen. Diese Idee ist die Basis der Entwicklung der so genannten weichen strukturvariablen Regelungen. Sie ermöglichen einen Kompromiss zwischen der linearen und der zeitoptimalen Lösung. Ihre Struktur und Funktionsweise werden in Abschnitt 3 erläutert.

In Abschnitt 4 wird gezeigt, wie beide Ansätze zusammengeführt werden können. Dies führt zum Hauptergebnis dieses Beitrags: ein neues Regelgesetz mit einer Regelgüte, die sehr nah an den zeitoptimalen Fall heranreichen kann. Gleichzeitig bleibt der Entwurf vergleichsweise einfach und der Stellgrößenverlauf ist stetig.

Schließlich werden die dargestellten Methoden in Abschnitt 5 anhand eines Beispiels verglichen.

\subsection{Vorbemerkungen}

Notation 1. Der Ausdruck $\mathbf{Z} \succ(\succeq) 0$ bedeutet, dass die Matrix $\mathbf{Z}$ symmetrisch und positiv (semi)definit ist. Ana$\log$ bedeutet $\mathbf{Z} \prec(\preceq) 0$, dass $\mathbf{Z}$ symmetrisch und negativ (semi)definit ist.

Notation 2. Der Ausdruck $\operatorname{conv} \mathcal{M}$ bezeichnet die konvexe Hülle der Menge $\mathcal{M}$.

Notation 3. Mit $\mathcal{G}(v, \rho) \subset \mathbb{R}^{n}$ wird die abegeschlossene Menge

$$
\mathcal{G}(v, \rho)=\left\{\mathbf{x} \in \mathbb{R}^{n} \mid v(\mathbf{x}) \leq \rho\right\},
$$

bezeichnet, wobei $\rho$ ein positiver Skalar und $v(\mathbf{x})$ eine positiv definite Funktion ist, d.h. $v(\mathbf{x})>0$ für $\mathbf{x} \neq \mathbf{0}$ und $v(\mathbf{0})=0$.

Notation 4. Mit $\mathcal{E}(\mathbf{R}, \rho) \subset \mathbb{R}^{n}$ wird das Ellipsoid

$$
\mathcal{E}(\mathbf{R}, \rho)=\left\{\mathbf{x} \in \mathbb{R}^{n} \mid \mathbf{x}^{\mathrm{T}} \mathbf{R} \mathbf{x} \leq \rho\right\}
$$

bezeichnet, wobei $\mathbf{R} \succ 0$ und $\rho$ ein positiver Skalar ist. Dies ist ein Spezialfall von Notation 3, wenn $v(\mathbf{x})=\mathbf{x}^{\mathrm{T}} \mathbf{R x}$ ist.

\section{Sättigende lineare Regler}

In diesem Abschnitt werden lineare Rückführungen betrachtet, die in die Sättigung gehen können. Folglich lautet das Regelgesetz

$$
u=\operatorname{sat}\left(u_{\mathrm{c}}\right)=\operatorname{sat}\left(-\mathbf{k}^{\mathrm{T}} \mathbf{x}\right),
$$

wobei die Sättigungsfunktion gegeben ist als

$$
\operatorname{sat}\left(u_{\mathrm{c}}\right)=\min \left(\left|u_{\mathrm{c}}\right|, u_{\max }\right) \cdot \operatorname{sgn}\left(u_{\mathrm{c}}\right) .
$$

Bei eintretender Sättigung wird das System nichtlinear. Deshalb macht die Tatsache, dass Sättigung auftreten kann, eine Anpassung der Stabilitätsanalyse notwendig. Die sättigende lineare Zustandsrückführung wird im Folgenden analysiert mit Hilfe der Methode von Ljapunov. Dafür wird folgende Definition benötigt:

Definition 1. Ein abgeschlossenes Gebiet $\mathcal{G}(v, \rho)$ heißt kontraktiv invariant, d.h., es bildet ein Einzugsgebiet für ein System $\dot{\mathbf{x}}=\boldsymbol{f}(\mathbf{x})$, wenn

$$
\frac{\partial v(\mathbf{x})}{\partial \mathbf{x}} \dot{\mathbf{x}}=\frac{\partial v(\mathbf{x})}{\partial \mathbf{x}} \boldsymbol{f}(\mathbf{x})<0
$$

für alle $\mathbf{x} \in \mathcal{G}(v, \rho) \backslash\{\mathbf{0}\}$ gilt. Somit ist $v(\mathbf{x})$ eine LjapunovFunktion des Systems.

Ausgehend von dieser Definition kann der folgende Satz formuliert werden: 
Satz 1 ([11]). Für ein dynamisches System

$$
\dot{\mathbf{x}}=\mathbf{A x}+\mathbf{b} u, \quad u=-\operatorname{sat}\left(\mathbf{f}^{\mathrm{T}} \mathbf{x}\right)
$$

sei ein Ellipsoid $\mathcal{E}(\mathbf{R}, \rho)$ gegeben, sodass

$$
\left(\mathbf{A}-\mathbf{b} \mathbf{f}^{\mathrm{T}}\right)^{\mathrm{T}} \mathbf{R}+\mathbf{R}\left(\mathbf{A}-\mathbf{b} \mathbf{f}^{\mathrm{T}}\right) \prec 0
$$

erfüllt ist. Dieses Ellipsoid ist genau dann kontraktiv invariant für das obige System, wenn eine Funktion $h(\mathbf{x})$ existiert, sodass $\mathcal{E}(\mathbf{R}, \rho)$ kontraktiv invariant unter der nichtsättigenden Rückführung $u=-h(\mathbf{x})$ ist, d. $h$.

$$
\begin{array}{ll}
|h(\mathbf{x})| \leq u_{\max } & \forall \mathbf{x} \in \mathcal{E}(\mathbf{R}, \rho), \\
\mathbf{x}^{\mathrm{T}} \mathbf{R}(\mathbf{A x}-\mathbf{b} h(\mathbf{x}))<0 & \forall \mathbf{x} \in \mathcal{E}(\mathbf{R}, \rho) \backslash\{\mathbf{0}\} .
\end{array}
$$

Die Kernaussage dieses Satzes ist, dass ein Ellipsoid für eine gesättigte Rückführung $\operatorname{sat}\left(\mathbf{f}^{\mathrm{T}} \mathbf{x}\right)$ kontraktiv invariant ist, wenn dieses Ellipsoid sowohl für die Rückführung $\mathbf{f}^{\mathrm{T}} \mathbf{x}$ ohne Berücksichtigung der Sättigung, wie auch für die nichtsättigende Rückführung $h(\mathbf{x})$ kontraktiv invariant ist. Dies bedeutet, dass diese beiden Rückführungen eine gemeinsame Ljapunov-Funktion $v(\mathbf{x})=\mathbf{x}^{\mathrm{T}} \mathbf{R} \mathbf{x}$ haben. Eine lineare Rückführung $h(\mathbf{x})=\mathbf{h}^{\mathrm{T}} \mathbf{x}$ ist in Satz 1 als Spezialfall enthalten. Für den Entwurf sättigender linearer Regler wird auf [11] verwiesen.

\section{Nichtsättigende nichtlineare Regler}

Alternativ zu sättigenden linearen Reglern ist es möglich, nichtlineare Regler zu betrachten, die nicht in die Sättigung gehen. Die schnellstmögliche Ausregelung eines Anfangszustands in den Ursprung ist durch die zeitoptimale Regelung gegeben. Das zeitoptimale Regelgesetz für ein lineares System mit Stellgrößenbeschränkung kann geschrieben werden als

$$
u=u_{\max } \operatorname{sgn}(\eta(\mathbf{x})),
$$

wobei $\eta$ die sogenannte Schaltfunktion ist. Es handelt sich dabei um eine nichtsättigende nichtlineare Rückführung, denn $u \leq u_{\max }$ ist jederzeit erfüllt. Sie weist jedoch einige Nachteile auf. Im Allgemeinen ist die Schaltfunktion $\eta(\mathbf{x})$ schwierig oder nicht analytisch ermittelbar. Des Weiteren können die Sprünge im Stellgrößenverlauf von realen Stellgliedern nicht geleistet werden.

Trotzdem ist es nützlich, den zeitoptimalen Verlauf zu betrachten. Da eine kürzere Ausregelzeit nicht möglich ist, kann dieser als Bezugsgröße verwendet werden, um die Regelgüte anderer Regler einzuschätzen.

\subsection{Weiche strukturvariable Regelung}

Wie bereits erwähnt, sind weiche strukturvariable Regler $(W S V R)^{1}$ nichtlineare Regler, die darauf ausgerichtet sind,

\footnotetext{
${ }^{1}$ Trotz des ähnlichen Namens sollte diese Klasse von Reglern nicht mit Gleitzustandsreglern (sliding-mode) verwechselt werden. Eine der inhärenten Eigenschaften von weichen strukturvariablen Regelungen ist, dass das System nicht in einen Gleitzustand übergehen kann.
}

den verfügbaren Stellgrößenbereich gut auszunutzen, um eine hohe Regelgüte $\mathrm{zu}$ erreichen. Eine Übersicht wird in [3] gegeben.

WSVR verwenden eine veränderliche lineare Rückführung, welche über einen Selektionsparameter $p$ vom aktuellen Zustand abhängt:

$$
u=-\mathbf{k}^{\mathrm{T}}(p(\mathbf{x})) \mathbf{x} \leq u_{\max }, \quad \forall t>t_{0}, \forall \mathbf{x}_{0} \in \mathcal{X}_{0} .
$$

Das Attribut strukturvariabel deutet an, dass die - ursprünglich lineare - Rückführung abhängig vom Zustand $\mathbf{x}$ verändert wird, während der Ausdruck weich aus der Tatsache herrührt, dass diese Veränderung stetig abläuft.

Der Selektionsparameter $p$ wird durch eine Selektionsstrategie

$$
S(\mathbf{x}, p, \dot{p}, \ldots)=0
$$

bestimmt. Diese Definition ist recht vielseitig, da Gl. (16) sowohl explizite und implizite Gleichungen als auch Differenzialgleichungen beinhaltet.

Die Selektionsstrategie $S$ und die Funktion $\mathbf{k}(p)$ müssen so gewählt werden, dass für alle möglichen Anfangsbedingungen die Stabilität garantiert ist, der Stellgrößenverlauf stetig ist und der Stellbereich gut ausgenutzt wird, ohne in die Sättigung zu gehen.

\subsection{WSVR mittels impliziter Ljapunov-Funktion}

Es existieren verschiedene Typen weicher strukturvariabler Regler. Im Folgenden gehen wir näher auf die weiche strukturvariable Regelung mittels impliziter LjapunovFunktion, kurz implizite WSVR [1;3;19], ein.

Es wird ohne Beschränkung der Allgemeinheit davon ausgegangen, dass die Regelstrecke in Regelungsnormalform vorliegt, d.h., sie hat die Gestalt $\dot{\mathbf{x}}=\mathbf{A x}+\mathbf{b} u$

$$
\mathbf{A}=\left[\begin{array}{cccc}
0 & 1 & \ldots & 0 \\
\vdots & \vdots & \ddots & \vdots \\
0 & 0 & \ldots & 1 \\
-a_{0} & -a_{1} & \ldots & -a_{n-1}
\end{array}\right], \quad \mathbf{b}=\left[\begin{array}{c}
0 \\
\vdots \\
0 \\
1
\end{array}\right]
$$

Um einen direkten Zusammenhang zwischen der Ausregelgeschwindigkeit des geschlossenen Regelkreises und des Selektionsparameters $p$ zu erhalten, wird der Regler $\mathbf{k}(p)$ so gewählt, dass die Pole $\lambda_{i}$ des geschlossenen Kreises mit kleiner werdendem $p$ auf Strahlen gemäß

$$
\lambda_{i}(p)=\frac{1}{p} \lambda_{i}(p=1)
$$

nach links verschoben werden. Dies kann durch die Wahl erreicht werden, wobei

$$
\mathbf{k}(p)=\mathbf{D}^{-1}(p) \hat{\mathbf{a}}-\mathbf{a},
$$

wobei

$$
\mathbf{D}(p)=\operatorname{diag}\left(p^{n}, p^{n-1}, \ldots, p\right), \quad p>0,
$$




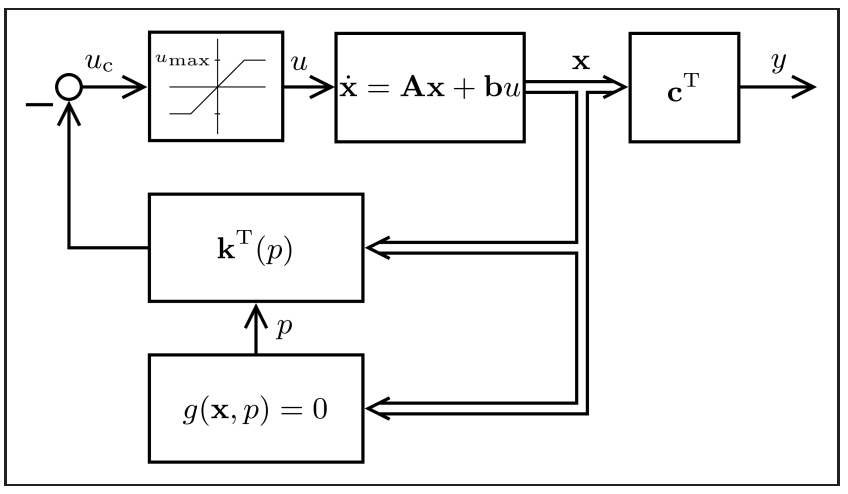

Bild 1: Strukturbild der impliziten WSVR.

gilt und $\mathbf{a}=\left[a_{0} \ldots a_{n-1}\right]^{\mathrm{T}}$ die Koeffizienten des charakteristischen Polynoms von A enthält.

Der Regler (19) führt zur Matrix des geschlossenen Kreises

$$
\hat{\mathbf{A}}(p)=\mathbf{A}-\mathbf{b k}^{\mathrm{T}}(p)=\mathbf{A}-\mathbf{b}\left(\mathbf{D}^{-1}(p) \hat{\mathbf{a}}-\mathbf{a}\right)^{\mathrm{T}},
$$

die unter Ausnutzung der besonderen Struktur der Regelungsnormalform geschrieben werden kann als

$$
\hat{\mathbf{A}}(p)=\frac{1}{p} \mathbf{D}(p) \hat{\mathbf{A}}_{1} \mathbf{D}^{-1}(p), \quad \hat{\mathbf{A}}_{1}=\hat{\mathbf{A}}(1) .
$$

Aus Gl. (22) lässt sich leicht ermitteln, dass die Eigenwerte von $\hat{\mathbf{A}}(p)$ in der Tat gegeben sind durch

$$
\lambda\{\hat{\mathbf{A}}(p)\}=\frac{1}{p} \lambda\left\{\hat{\mathbf{A}}_{1}\right\} .
$$

Folglich wird der geschlossene Kreis schneller für kleinere $p$, wenn â so gewählt wird, dass die Eigenwerte von $\hat{\mathbf{A}}_{1}$ in der offenen linken Halbebene liegen. Der Vektor $\hat{\mathbf{a}}=\left[\hat{a}_{0} \ldots \hat{a}_{n-1}\right]^{\mathrm{T}}$ enthält die Koeffizienten des charakteristischen Polynoms von $\hat{\mathbf{A}}_{1}$. Da die Eigenwerte von $\hat{\mathbf{A}}_{1}$ durch geeignete Wahl von â beliebig platziert werden können, kann man ohne Einschränkung der Allgemeinheit annehmen, dass $p \in \mathcal{P}=(0,1]$ gilt.

Die Auswahlstrategie impliziter WSVR [1;3] gibt den Parameter $p$ so vor, dass er während des Ausregelverlaufs fällt. Sie ist durch die implizite Gleichung

$$
g(\mathbf{x}, p)=e(p) \mathbf{x}^{\mathrm{T}} \mathbf{R}(p) \mathbf{x}-1=0
$$

gegeben, wobei

$$
\mathbf{R}(p)=\mathbf{D}^{-1}(p) \mathbf{R}_{1} \mathbf{D}^{-1}(p)
$$

ist. Die skalare Funktion $e(p)$ ist ein Polynom in $p$ mit Koeffizienten, die von $\mathbf{x}$ abhängen, und lautet

$$
e(p)=\frac{\mathbf{k}^{\mathrm{T}}(p) \mathbf{R}^{-1}(p) \mathbf{k}(p)}{u_{\max }^{2}}=\sum_{i=0}^{2 n} c_{i}(\mathbf{x}) p^{i} .
$$

Die Funktion $e(p)$ ist so gewählt, dass sich aus der Auswahlstrategie (24) ein Regler $\mathbf{k}(p)$ ergibt, welcher den Stellbereich gut ausnutzt und die Beschränkung immer einhält, d.h., es gilt immer $\left|\mathbf{k}(p)^{\mathrm{T}} \mathbf{x}\right| \leq u_{\max }$.
Die Struktur des geschlossenen Regelkreises ist in Bild 1 dargestellt. Folgender Satz beantwortet die Frage nach seiner Stabilität.

Satz 2 ([1;3]). Gegeben sei eine lineare Regelstrecke in Regelungsnormalform mit der Zustandsrückführung (19) und der Selektionsstrategie (24). Wenn die Ungleichungen

$$
\begin{aligned}
& \mathbf{R}_{1} \succ 0, \\
& \hat{\mathbf{A}}_{1}^{\mathrm{T}} \mathbf{R}_{1}+\mathbf{R}_{1} \hat{\mathbf{A}}_{1} \prec 0, \\
& e^{\prime}(p) \mathbf{R}_{1}+\frac{e(p)}{p}\left(\mathbf{N R}_{1}+\mathbf{R}_{1} \mathbf{N}\right) \prec 0
\end{aligned}
$$

für alle $p \in \mathcal{P}=(0,1]$ erfüllt sind, wobei

$$
\mathbf{N}=\operatorname{diag}(-n, \ldots,-1),
$$

dann hat die Selektionsstrategie (24) eine eindeutige Lösung auf dem Intervall $\mathcal{P}$ für alle $\mathbf{x}$ in der Menge

$$
\mathcal{E}_{1} \backslash\{\mathbf{0}\}, \quad \mathcal{E}_{1}=\mathcal{E}\left(e(1) \mathbf{R}_{1}, 1\right) .
$$

Außerdem ist $v(\mathbf{x})=p(\mathbf{x})$ eine durch Gl. (24) gegebene implizite Ljapunov-Funktion [2] des Regelkreises auf $\mathcal{E}_{1}$.

Um sicherzustellen, dass das Regelgesetz für alle möglichen $\mathbf{x}_{0} \in \mathcal{X}_{0}$ gültig ist, muss $\mathcal{X}_{0} \subseteq \mathcal{E}_{1}$ erfüllt sein. Wenn $\mathcal{X}_{0}$ als konvexes Polyeder mit $N$ Eckpunkten $\mathbf{v}_{j}$ gegeben ist, entspricht diese Nebenbedingung den $N$ Ungleichungen

$$
g\left(\mathbf{v}_{j}, 1\right)=e(1) \mathbf{v}_{j}^{\mathrm{T}} \mathbf{R}_{1} \mathbf{v}_{j}-1<0 \quad \forall j=1, \ldots, N .
$$

Bemerkung 1. Dadurch, dass die implizite Gleichung (24) genau eine Lösung auf dem Intervall $(0,1]$ hat, kann diese mittels eines Bisektionsverfahrens sehr schnell, einfach und in jedem Fall bestimmt werden.

Bemerkung 2. Die Tatsache, dass $v(\mathbf{x})=p(\mathbf{x})$ eine Ljapunov-Funktion auf $\mathcal{E}_{1}$ ist, stellt sicher, dass $\mathcal{E}_{1}$ kontraktiv invariant ist. Außerdem ist garantiert, dass $p$ mit der Zeit stetig fällt. Die Regelung wird dadurch immer schneller, was der Grund für die gute Regelgüte ist.

Bemerkung 3. Das Regelgesetz enthält Terme $p^{-n}$, die zu numerischen Problemen für kleine Werte von $p$ führen können. Deshalb wird ein Minimalwert $p_{\min }>0$ festgelegt. Sobald dieser Wert erreicht wird, verändert sich der Regler nicht mehr und arbeitet linear als $\mathbf{k}\left(p_{\min }\right)$. Die implizite Ljapunov-Funktion kann mit einer quadratischen Funktion stetig erweitert werden zu

$$
v(\mathbf{x})=\left\{\begin{array}{ll}
p(\mathbf{x}) & g\left(\mathbf{x}, p_{\min }\right)<0 \\
p_{\min } e\left(p_{\min }\right) \mathbf{x}^{\mathrm{T}} \mathbf{R}\left(p_{\min }\right) \mathbf{x} & g\left(\mathbf{x}, p_{\min }\right) \geq 0
\end{array} .\right.
$$

Diese Ljapunov-Funktion ist stetig, aber nicht differenzierbar auf $e\left(p_{\min }\right) \mathbf{x}^{\mathrm{T}} \mathbf{R}\left(p_{\min }\right) \mathbf{x}=1$. In $[1 ; 16]$ wird dargelegt, wie man mittels Dini-Derivierter auch stellenweise nicht differenzierbare Ljapunov-Funktionen verwenden kann. Die 
Wahl von $p_{\text {min }}$ hängt wesentlich von der Systemordnung $n$ ab. Typische Werte liegen zwischen 0,01 und 0,1.

Bemerkung 4. Wie bereits erwähnt stellt die Annahme, dass die Regelstrecke in Regelungsnormalform vorliegt, keine Beschränkung der Allgemeinheit dar. Wenn nämlich die Strecke vollständig steuerbar ist, kann sie immer mittels einer regulären Transformation in die Form (17) gebracht werden. Die Reglerparameter $\mathbf{k}(p)$ und $\mathbf{R}(p)$ müssen dann zur Regelung in die Originalkoordinaten rücktransformiert werden.

\subsection{Entwurf impliziter WSVR}

\subsubsection{Entwurf durch konvexe Optimierung}

Der Entwurf einer impliziten WSVR besteht in der Wahl geeigneter Werte für die Parameter $\mathbf{a}$ und $\mathbf{R}_{1}$. Sie müssen sich innerhalb des durch die Ungleichungen (27) und (30) definierten zulässigen Raums befinden und so gewählt werden, dass ein gutes Regelverhalten erreicht wird. Die Optimierung kann mit einem beliebigen Suchverfahren wie z. B. mit einer Evolutionsstrategie erfolgen. Diese können aber unter Umständen sehr zeitaufwändig sein.

Die Entwurfszeit lässt sich deutlich verringern, indem das Optimierungsproblem in ein konvexes Optimierungsproblem umgeformt wird. Zu diesem Zweck werden die Ungleichungen (27) und (30) in lineare Matrixungleichungen (LMIs) [5] in den Variablen

$$
\mathbf{Q}=\mathbf{R}_{1}^{-1} \text { und } \mathbf{z}=\mathbf{R}_{1}^{-1} \hat{\mathbf{a}}
$$

transformiert. Zur Abkürzung wird

$$
\mathbf{A}_{0}=\mathbf{A}+\mathbf{b a}^{\mathrm{T}}
$$

sowie

$$
\mathbf{P}(l, k)= \begin{cases}\prod_{q=0}^{k-1}[(l-q) \mathbf{I}+\mathbf{M}] & \text { für } k \neq 0 \\ \mathbf{I} & \text { für } k=0\end{cases}
$$

eingeführt, wobei $\mathbf{M}=\operatorname{diag}(0,1, \ldots, n-1)$. Satz 2 lässt sich dann folgendermaßen in LMI-Form überführen:

Satz 3 ([19]). Gegeben seien ein lineares System in Regelungsnormalform, der Regler (19) und die Selektionsstrategie (24). Mit $m$ wird die Ordnung des Polynoms $e^{\prime}(p)$ bezeichnet. Wenn die LMIs

$$
\begin{aligned}
& \mathbf{Q} \succ 0, \\
& \mathbf{Q} \mathbf{A}_{0}^{\mathrm{T}}+\mathbf{A}_{0} \mathbf{Q}-\mathbf{z b}^{\mathrm{T}}-\mathbf{b} \mathbf{z}^{\mathrm{T}} \prec 0, \\
& \mathbf{Q N}+\mathbf{N Q} \prec 0,
\end{aligned}
$$

und, für alle $i \in\{0,1, \ldots\}$,

$$
\sum_{k=0}^{i}\left(\begin{array}{l}
i \\
k
\end{array}\right) \mathbf{a}^{\mathrm{T}} \mathbf{P}(0, i-k) \mathbf{Q N P}(0, k) \mathbf{a}-\mathbf{z}^{\mathrm{T}} \mathbf{N P}(n, i) \mathbf{a} \geq 0 .
$$

erfüllt sind, dann hat die Selektionsstrategie (24) eine eindeutige Lösung auf dem Intervall $\mathcal{P}$ für alle $\mathbf{x}$ in der
Menge $\mathcal{E}_{1} \backslash\{\mathbf{0}\}$. Darüber hinaus ist $v(\mathbf{x})=p(\mathbf{x})$ eine implizite Ljapunov-Funktion des geschlossenen Regelkreises auf $\mathcal{E}_{1}$.

Da die Matrix $\mathbf{Q}$ beliebig skalierbar ist, lässt sich die Ungleichung (30) ebenfalls als LMI in der Form

$$
\begin{aligned}
& {\left[\begin{array}{cc}
1 & \mathbf{v}_{j}^{\mathrm{T}} \\
\mathbf{v}_{j} & \mathbf{Q}
\end{array}\right] \succ 0,} \\
& {\left[\begin{array}{cc}
u_{\max }^{2}-\mathbf{a}^{\mathrm{T}} \mathbf{Q} \mathbf{a}+2 \mathbf{a}^{\mathrm{T}} \mathbf{z} & \mathbf{z}^{\mathrm{T}} \\
\mathbf{z} & \mathbf{Q}
\end{array}\right] \succ 0,}
\end{aligned}
$$

schreiben [19]. Dann ist $\mathcal{X}_{0} \subseteq \mathcal{E}_{1}$ sichergestellt.

Bemerkung 5. Die Bedingungen (35) sind hinreichend, aber nicht notwendig für das Einhalten von (27). Demnach ist die Lösungsmenge der Ungleichungen (35) eine Teilmenge der Lösungsmenge der Ungleichungen (27).

Für die Optimierung ist noch eine konvexe Gütefunktion zu wählen. Da keine direkte konvexe Abhängigkeit zwischen der Regelgüte und den Regelparametern existiert, kann diese nur indirekt über ein konvexes Gütemaß beeinflusst werden.

Man kann beispielsweise zuerst einen guten linearen Regler k(1) wählen, aus dem sich â ergibt, und daraufhin das Volumen des Anfangsgebiets $\mathcal{E}_{1}$ maximieren, indem $\mathbf{Q}$ optimiert wird $[3 ; 19]$. Eine weitere Möglichkeit ist dadurch gegeben, die Abklingrate des mit dem Regler k(1) geregelten Systems, d. h. des Systems $\dot{\mathbf{x}}=\hat{\mathbf{A}}_{1} \mathbf{x}$, zu maximieren. In diesem Fall sind sowohl $\mathbf{z}$ als auch $\mathbf{Q}$ Optimierungsvariablen.

Definition 2 ([5]). Die Abklingrate eines Systems $\dot{\mathbf{x}}=\hat{\mathbf{A}} \mathbf{x}$ ist definiert als das größte $\delta$, sodass

$$
\lim _{t \rightarrow \infty} e^{\delta t}\|\mathbf{x}(t)\|=0
$$

für alle Trajektorien $\mathbf{x}(t)$ gilt.

Wenn für ein bestimmtes $\delta$ eine Matrix $\mathbf{Q}$ existiert, sodass die LMI

$$
\mathbf{Q} \hat{\mathbf{A}}^{\mathrm{T}}+\hat{\mathbf{A}} \mathbf{Q} \preceq-2 \delta \mathbf{Q}
$$

erfüllt ist, ist $\delta$ eine untere Abschätzung der Abklingrate [5]. Wenn diese untere Abschätzung maximiert werden soll, lautet das Optimierungsproblem in den Variablen $\delta, \mathbf{z}$ und $\mathbf{Q}$ folglich

$$
\begin{aligned}
& \max \delta \\
& \text { sodass } \\
& \mathbf{Q A}_{0}^{\mathrm{T}}+\mathbf{A}_{0} \mathbf{Q}-\mathbf{z b}^{\mathrm{T}}-\mathbf{b} \mathbf{z}^{\mathrm{T}} \prec-2 \delta \mathbf{Q}, \\
& \text { (35a), (35c), (35d), (36a) und (36b). }
\end{aligned}
$$

Im Optimierungsproblem (39) ist $\delta$ eine Variable. Die Ungleichung (39b) ist deshalb aufgrund des Produktterms $\delta \mathbf{Q}$ keine LMI. Das Problem ist jedoch quasikonvex in $\delta$ [5]. Deshalb kann es mittels eines Bisektionsverfahrens bezüglich $\delta$ gelöst werden. In jedem Iterationsschritt wird dann für ein bestimmtes $\delta$ eine gültige Lösung für die obigen 
LMIs gesucht. Der maximale Wert für $\delta$, für den eine gültige Lösung $\mathbf{Q}$ und $\mathbf{z}$ existiert, entspricht dann dem Optimum.

\subsubsection{Entwurf mittels Suchverfahren}

Das Optimierungsproblem (39) kann zwar schnell gelöst werden, der gefundene Regler liefert jedoch aus zwei Gründen nicht das schnellstmögliche Ergebnis. Erstens ist, wie in Bemerkung 5 erwähnt, die Lösungsmenge der Nebenbedingungen (35) nur eine Teilmenge der Lösungsmenge der Nebenbedingungen (27). Zweitens kann die Ausregelzeit nur indirekt optimiert werden, weil man auf konvexe Gütemaße angewiesen ist.

Ein Optimierungsproblem mit einem beliebigen Gütemaß und den Nebenbedingungen (27) und (30) ermöglicht bessere Ergebnisse. Insbesondere können solche Gütemaße verwendet werden, welche die tatsächliche Performance abbilden. Diese kann beispielsweise durch Simulationen ermittelt werden. Da ein solches Optimierungsproblem jedoch nicht konvex ist, kann es nur mittels Suchverfahren, beispielsweise mittels Evolutionsstrategien, gelöst werden. Die besseren Ergebnisse erkauft man sich deshalb mit deutlich längeren Rechenzeiten [19].

Damit die Optimierung mittels Suchverfahren mit einem zulässigen, guten Wert beginnt, ist es sinnvoll, die Lösung des konvexen Optimierungsproblems als Startwert zu verwenden.

\section{Sättigende nichtlineare Regler}

Weiche strukturvariable Regler sind darauf ausgerichtet, den Stellgrößenbereich möglichst gut auszunutzen. Sie gehen jedoch nie in den Bereich der Sättigung und können deshalb die Stellgrößenbeschränkung $u_{\max }$ nur punktweise erreichen. Diese Eigenschaft steht einer noch schnelleren Regelung im Weg. Wenn die Stabilität garantiert werden kann, gibt es im Allgemeinen keinen Grund, warum weiche strukturvariable Regler nicht im gesättigten Bereich arbeiten dürften.

Satz 1 sagt aus, dass ein System mit linearer, gesättigter Rückführung stabil ist, wenn eine nichtsättigende stabile Rückführung existiert, sodass beide Rückführungen eine gemeinsame quadratische Ljapunov-Funktion haben. Dieser Satz kann erweitert werden, sodass er auch auf den Fall nichtlinearer Rückführungen und anderer LjapunovFunktionen zutrifft:

Satz 4. Gegeben seien das System

$$
\dot{\mathbf{x}}=\mathbf{A x}+\mathbf{b} u, \quad u=-\operatorname{sat}(f(\mathbf{x})),
$$

eine positiv definite Funktion $v(\mathbf{x})$ und eine Menge $\mathcal{G}(v, \rho)$, sodass

$$
\frac{\partial v}{\partial \mathbf{x}}(\mathbf{A x}-\mathbf{b} f(\mathbf{x}))<0 \quad \forall \mathbf{x} \in \mathcal{G}(v, \rho) \backslash\{\mathbf{0}\}
$$

gilt. Die Menge $\mathcal{G}(v, \rho)$ ist genau dann kontraktiv invariant für das obige System, wenn eine Funktion $h(\mathbf{x})$ existiert, sodass $\mathcal{G}(v, \rho)$ kontraktiv invariant unter der Rückführung $u=-h(\mathbf{x})$ ist, $d . h$.

$$
\begin{array}{ll}
|h(\mathbf{x})| \leq u_{\max } & \forall \mathbf{x} \in \mathcal{G}(v, \rho), \\
\frac{\partial v}{\partial \mathbf{x}}(\mathbf{A x}-\mathbf{b} h(\mathbf{x}))<0 & \forall \mathbf{x} \in \mathcal{G}(v, \rho) \backslash\{\mathbf{0}\} .
\end{array}
$$

Beweis. Die Notwendigkeit ist offensichtlich: Wenn eine Rückführung $f(\mathbf{x})$ mit den obigen Eigenschaften existiert, dann muss ebenfalls eine passende Funktion $h(\mathbf{x})$ existieren. Ein immer gültiges Beispiel einer solchen Funktion ist $h(\mathbf{x})=\operatorname{sat}(f(\mathbf{x}))$.

Es folgt der an den Beweis von Satz 1 aus [11] angelehnte Nachweis, dass die Voraussetzungen auch hinreichend sind. Aus der Eigenschaft (42) kann man folgern, dass

$$
u=-\operatorname{sat}(f(\mathbf{x})) \in \operatorname{conv}\{-h(\mathbf{x}),-f(\mathbf{x})\}
$$

und somit auch

$$
\dot{\mathbf{x}} \in \operatorname{conv}\{\mathbf{A x}-\mathbf{b} h(\mathbf{x}), \mathbf{A x}-\mathbf{b} f(\mathbf{x})\}
$$

gilt. Wenn man nun die zeitliche Ableitung der Funktion $v(\mathbf{x})$, gegeben durch

$$
\frac{\mathrm{d} v(\mathbf{x})}{\mathrm{d} t}=\frac{\partial v}{\partial \mathbf{x}} \dot{\mathbf{x}}
$$

betrachtet, ergibt sich

$$
\frac{\partial v}{\partial \mathbf{x}} \dot{\mathbf{x}} \in \operatorname{conv}\left\{\frac{\partial v}{\partial \mathbf{x}}(\mathbf{A x}-\mathbf{b} h(\mathbf{x})), \frac{\partial v}{\partial \mathbf{x}}(\mathbf{A x}-\mathbf{b} f(\mathbf{x}))\right\},
$$

woraus

$$
\frac{\partial v}{\partial \mathbf{x}} \dot{\mathbf{x}} \leq \max \left\{\frac{\partial v}{\partial \mathbf{x}}(\mathbf{A x}-\mathbf{b} h(\mathbf{x})), \frac{\partial v}{\partial \mathbf{x}}(\mathbf{A x}-\mathbf{b} f(\mathbf{x}))\right\}
$$

folgt. Unter Verwendung von (41) und (43) bedeutet dies, dass

$$
\frac{\partial v}{\partial \mathbf{x}} \dot{\mathbf{x}}<0 \quad \forall \mathbf{x} \in \mathcal{G}(v, \rho) \backslash\{\mathbf{0}\}
$$

gilt. Die Funktion $v$ ist demnach eine Ljapunov-Funktion für das gesättigte System. Deshalb ist $\mathcal{G}(v, \rho)$ kontraktiv invariant für das System unter der Rückführung $u=$ $-\operatorname{sat}(f(\mathbf{x}))$.

\subsection{Sättigende implizite WSVR}

Wir können nun Satz 4 anwenden, um die implizite WSVR aus Abschnitt 3.2 weiter zu verbessern. Die nichtsättigende stabilisierende Rückführung ist bereits bekannt aus Gl. (19):

$$
h(\mathbf{x})=\left(\mathbf{D}^{-1}(p) \hat{\mathbf{a}}-\mathbf{a}\right)^{\mathrm{T}} \mathbf{x} .
$$

Die Funktion $v(\mathbf{x})=p(\mathbf{x})$ ist eine Ljapunov-Funktion für diese Rückführung. Dies bedeutet nach Satz 4, dass eine neue Rückführung $u=-\operatorname{sat}(f(\mathbf{x}))$ das System garantiert stabilisiert, wenn $v$ eine gültige Ljapunov-Funktion für die Rückführung $u=-f(\mathbf{x})$ ist. Der folgende Satz stellt eine solche Rückführung vor: 
Satz 5. Es wird das System $\dot{\mathbf{x}}=\mathbf{A x}+\mathbf{b} u$ mit der Rückführung

$$
u=-\operatorname{sat}\left(\left(\mathbf{k}^{\star}(p)\right)^{\mathrm{T}} \mathbf{x}\right), \quad \mathbf{k}^{\star}(p)=\mathbf{D}^{-1}(p) \hat{\mathbf{a}}^{\star}-\mathbf{a}
$$

und der Selektionsstrategie

$$
g(\mathbf{x}, p)=e(p) \mathbf{x}^{\mathrm{T}} \mathbf{R}(p) \mathbf{x}-1=0,
$$

siehe Gl. (24), betrachtet. Wenn die Bedingungen

$$
\begin{aligned}
& \mathbf{R}_{1} \succ 0, \\
& \hat{\mathbf{A}}_{1}^{\mathrm{T}} \mathbf{R}_{1}+\mathbf{R}_{1} \hat{\mathbf{A}}_{1} \prec 0, \\
& e^{\prime}(p) \mathbf{R}_{1}+\frac{e(p)}{p}\left(\mathbf{N R}_{1}+\mathbf{R}_{1} \mathbf{N}\right) \prec 0, \\
& \hat{\mathbf{A}}_{1}^{\star \mathrm{T}} \mathbf{R}_{1}+\mathbf{R}_{1} \hat{\mathbf{A}}_{1}^{\star} \prec 0
\end{aligned}
$$

erfüllt sind, wobei

$$
\begin{aligned}
\hat{\mathbf{A}}_{1} & =\mathbf{A}-\mathbf{b}(\hat{\mathbf{a}}-\mathbf{a})^{\mathrm{T}}, \\
\hat{\mathbf{A}}_{1}^{\star} & =\mathbf{A}-\mathbf{b}\left(\hat{\mathbf{a}}^{\star}-\mathbf{a}\right)^{\mathrm{T}}, \\
\mathbf{N} & =\operatorname{diag}(-n, \ldots,-1)
\end{aligned}
$$

gilt, dann stabilisiert die Rückführung (49) das System für alle $\mathbf{x} \in \mathcal{E}_{1}=\mathcal{E}\left(e(1) \mathbf{R}_{1}, 1\right)$.

Beweis. Nach Satz 2 garantieren die ersten drei Nebenbedingungen (50a) bis (50c), dass $u=-h(\mathbf{x}), h(\mathbf{x})$ wie in Gl. (48), eine nichtsättigende stabilisierende Rückführung mit der impliziten Ljapunov-Funktion $v(\mathbf{x})=p(\mathbf{x})$ auf $\mathcal{E}_{1}$ ist. Deshalb folgt mittels Satz 4, dass die Rückführung (49) das System auf $\mathcal{E}_{1}$ stabilisiert, wenn

$$
\frac{\partial v}{\partial \mathbf{x}} \dot{\mathbf{x}}=\frac{\partial p}{\partial \mathbf{x}}\left(\mathbf{A}-\mathbf{b}\left(\mathbf{k}^{\star}(p)\right)^{\mathrm{T}}\right) \mathbf{x}<0
$$

für alle $\mathbf{x} \in \mathcal{E}_{1} \backslash\{\boldsymbol{0}\}$ erfüllt ist. Da der Regler $\mathbf{k}^{\star}(p)$ die gleiche Struktur hat wie der Regler $\mathbf{k}(p)$, kann die Matrix des geschlossenen Kreises unter der Rückführung (49) analog zu Gl. (22) umgeformt werden:

$$
\mathbf{A}-\mathbf{b}\left(\mathbf{k}^{\star}(p)\right)^{\mathrm{T}}=\hat{\mathbf{A}}^{\star}(p)=\frac{1}{p} \mathbf{D}(p) \hat{\mathbf{A}}_{1}^{\star} \mathbf{D}^{-1}(p) .
$$

Nach der Methode der impliziten Differentiation ist

$$
\frac{\partial p}{\partial \mathbf{x}}=-\frac{\partial g(p, \mathbf{x}) / \partial \mathbf{x}}{\partial g(p, \mathbf{x}) / \partial p},
$$

womit sich zusammen mit Gl. (55) nach einigen Umformschritten aus Gl. (54)

$$
-\frac{\mathbf{x}\left(\hat{\mathbf{A}}^{\star}(p)^{\mathrm{T}} \mathbf{R}(p)+\mathbf{R}(p) \hat{\mathbf{A}}^{\star}(p)\right) \mathbf{x}}{e(p)^{-1} \partial g(p, \mathbf{x}) / \partial p}<0
$$

ergibt. Der Nenner ist unabhängig von der verwendeten Rückführung und somit wie bei der nichtsättigenden impliziten WSVR für alle $\mathbf{x} \in \mathcal{E}_{1}$ und $p \in \mathcal{P}$ negativ [3]. Der Gesamtausdruck ist folglich negativ, wenn die Matrix

$$
\hat{\mathbf{A}}^{\star}(p)^{\mathrm{T}} \mathbf{R}(p)+\mathbf{R}(p) \hat{\mathbf{A}}^{\star}(p)
$$

negativ definit ist. Da für reguläre Matrizen $\mathbf{X}$ der Zusammenhang $\mathbf{X}^{\mathrm{T}} \mathbf{Z X} \prec 0 \Leftrightarrow \mathbf{Z} \prec 0$ gilt [5], können die von $p$ abhängigen Terme eliminiert werden. Man erhält als Bedingung für Stabilität die LMI

$$
\hat{\mathbf{A}}_{1}^{\star \mathrm{T}} \mathbf{R}_{1}+\mathbf{R}_{1} \hat{\mathbf{A}}_{1}^{\star} \prec 0 .
$$

Diese Bedingung ist nach Voraussetzung (50d) erfüllt.

Auch hier gilt Bemerkung 2: Die Menge $\mathcal{E}_{1}$ ist kontraktiv invariant und $p$ fällt mit der Zeit.

Bemerkung 6. Da das Auswahlgesetz (24) unverändert beibehalten wird, bleibt der Online-Rechenaufwand ebenfalls unverändert. Der Entwurfsaufwand erhöht sich durch die zusätzliche Variable $\mathbf{a}^{\star}$ zwar leicht, dafür werden aber deutlich schnellere Regelvorgänge ermöglicht.

Bemerkung 7. Der Regler kann auch mit einem Beobachter verwendet werden. Der in [4] vorgestellte Stabilitätssatz ist auch für den hier vorgestellten Regler mit Beobachter gültig.

\subsection{Entwurf sättigender impliziter WSVR}

Für den Entwurf wird wie in Abschnitt 3.3 auf Methoden der konvexen Optimierung zurückgegriffen. Analog zu Satz 3 gibt folgender Satz die Nebenbedingungen in LMIForm an:

Satz 6. Gegeben seien ein lineares System in Regelungsnormalform, der Regler (49) und die Selektionsstrategie (24). Wenn die LMIs (35) und

$$
\mathbf{Q} \mathbf{A}_{0}^{\mathrm{T}}+\mathbf{A}_{0} \mathbf{Q}-\mathbf{z}^{\star} \mathbf{b}^{\mathrm{T}}-\mathbf{b}\left(\mathbf{z}^{\star}\right)^{\mathrm{T}} \prec 0
$$

erfüllt sind, wobei $\mathbf{z}^{\star}=\mathbf{R}_{1}^{-1} \hat{\mathbf{a}}^{\star}$ und $\mathbf{A}_{0}=\mathbf{A}+\mathbf{b a}^{\mathrm{T}}$, dann hat die Selektionsstrategie (24) eine eindeutige Lösung auf dem Intervall $\mathcal{P}$ für alle $\mathbf{x}$ in der Menge $\mathcal{E}_{1} \backslash\{\mathbf{0}\}$. Darüber hinaus ist $v(\mathbf{x})=p(\mathbf{x})$ eine implizite Ljapunov-Funktion des geschlossenen Regelkreises auf $\mathcal{E}_{1}$.

Beweis. Die Nebenbedingungen (50a) bis (50c) aus Satz 5 entsprechen exakt den Nebenbedingungen (27) aus Satz 2. Die zugehörigen LMIs (35) können deshalb unverändert übernommen werden. Die zusätzliche Nebenbedingung (50d) kann analog zu Gleichung (27b) in LMI-Form gebracht werden. Durch beidseitige Multiplikation von $\mathbf{Q}$ an (50d) und Einführen der zusätzlichen Variablen $\mathbf{z}^{\star}$ gelangt man zur LMI (60).

Da die Nebenbedingung $\mathcal{X}_{0} \subseteq \mathcal{E}_{1}$ nur von der nichtsättigenden Rückführung abhängig ist, können die LMIs (36) ohne Änderungen beibehalten werden.

Das konvexe Gütemaß sollte angepasst werden und sich auf den Regler $\mathbf{k}^{\star}(p)$ beziehen, denn dieser wird für die Regelung verwendet und ist daher maßgeblich. Vom Regler $\mathbf{k}(p)$ fordert man lediglich Stabilität. Um gute Ergebnisse zu erreichen, muss allerdings Folgendes beachtet werden: Der Regler $\mathbf{k}^{\star}(p)$ wird nur durch die Nebenbedingung begrenzt, dass er eine gemeinsame Ljapunov-Funktion mit dem nichtsättigenden Regler $\mathbf{k}(p)$ haben muss. Dies kann 
dazu führen, dass die Einträge von $\hat{\mathbf{a}}^{\star}$ sehr groß werden. Sehr hohe Verstärkungen bringen aufgrund der Sättigung kaum eine Verbesserung der Regelgüte. Dadurch, dass der Bereich zulässiger $\mathbf{a}^{\star}$ nicht ausreichend beschränkt ist, können aber numerische Probleme bei der Optimierung auftreten. Außerdem sind zu hohe Verstärkungen unerwünscht, weil dadurch das Rauschen unnötig verstärkt wird. Eine zusätzliche virtuelle Stellgrößenbeschränkung

$$
\left|\mathbf{k}^{\star \mathrm{T}}(1) \mathbf{x}\right| \leq \beta u_{\max } \quad \forall \mathbf{x} \in \mathcal{E}_{1}, \quad \beta \gg 1
$$

verhindert dieses Phänomen [7].

Für die sättigende implizite WSVR haben sich die besten Ergebnisse ergeben, wenn die Abklingrate des Systems $\dot{\mathbf{x}}=\hat{\mathbf{A}}_{1}^{\star} \mathbf{x}$ als Gütemaß gewählt wird. Wenn das Optimierungsproblem (39) um die LMI (60) erweitert wird und die obigen Anmerkungen eingearbeitet werden, erreicht man das folgende LMI-basierte Optimierungsproblem:

$$
\begin{aligned}
& \max \delta \\
& \text { sodass } \\
& \mathbf{Q A}_{0}^{\mathrm{T}}+\mathbf{A}_{0} \mathbf{Q}-\mathbf{z b}^{\mathrm{T}}-\mathbf{b} \mathbf{z}^{\mathrm{T}} \prec 0, \\
& \mathbf{Q A}_{0}^{\mathrm{T}}+\mathbf{A}_{0} \mathbf{Q}-\mathbf{z}^{\star} \mathbf{b}^{\mathrm{T}}-\mathbf{b}\left(\mathbf{z}^{\star}\right)^{\mathrm{T}} \prec-2 \delta \mathbf{Q}, \\
& {\left[\begin{array}{cc}
\beta^{2} u_{\max }^{2}-\mathbf{a}^{\mathrm{T}} \mathbf{Q} \mathbf{a}+2 \mathbf{a}^{\mathrm{T}} \mathbf{z}^{\star} & \left(\mathbf{z}^{\star}\right)^{\mathrm{T}} \\
\mathbf{z}^{\star} & \mathbf{Q}
\end{array}\right] \succ 0,} \\
& (35 \mathrm{a}),(35 \mathrm{c}),(35 \mathrm{~d}),(36 \mathrm{a}) \text { und }(36 \mathrm{~b}) .
\end{aligned}
$$

Die Variablen sind $\delta, \mathbf{Q}=\mathbf{R}_{1}^{-1}, \mathbf{z}=\mathbf{R}_{1}^{-1} \hat{\mathbf{a}}$ und $\mathbf{z}^{\star}=\mathbf{R}_{1}^{-1} \hat{\mathbf{a}}^{\star}$. Der Parameter $\beta$ muss vor dem Entwurf festgelegt werden.

Analog zu Abschnitt 3.3.2 ist auch für die sättigende implizite WSVR eine Optimierung mittels eines Suchverfahrens, wie beispielsweise einer Evolutionsstrategie, auf Basis der Nebenbedingungen (50), (30) und eines beliebigen Gütemaßes möglich.

\section{Beispiel: Tiefenregelung eines U-Boots}

Als Beispiel wird die Regelung der Tauchtiefe eines UBoots betrachtet. Diese Regelstrecke stammt ursprünglich aus [10] und wurde in [3] als Beispiel für WSVR verwendet. Die Regelstrecke ist gegeben durch

$$
\begin{aligned}
& \dot{\mathbf{x}}=\left[\begin{array}{ccc}
0 & 1 & 0 \\
0 & 0 & 1 \\
0 & 0 & -0,005
\end{array}\right] \mathbf{x}+\left[\begin{array}{l}
0 \\
0 \\
1
\end{array}\right] \operatorname{sat}\left(u_{\mathrm{c}}\right), \\
& y=x_{1},
\end{aligned}
$$

wobei $y$ die Tauchtiefe ist. Dabei gilt $u_{\max }=2,5 \cdot 10^{-5}$ und $\mathcal{X}_{0}=\left\{\mathbf{x}|| x_{1}|\leq 10,| x_{2}|\leq 0,05,| x_{3} \mid \leq 0,0046\right\}$.

Mit den im Anhang angegebenen Parametern aus der konvexen Optimierung ergeben sich für den Anfangszustand $\mathbf{x}_{0}^{\mathrm{T}}=\left[\begin{array}{lll}0 & 0 & -0,004\end{array}\right]$ die in Bild 2 und Bild 3 dargestellten Zeitverläufe. Die sättigende implizite WSVR erreicht nach der zeitoptimalen Regelung die schnellste Ausregelzeit.

Im Folgenden demonstrieren wir das Potenzial der sättigenden impliziten WSVR durch eine Optimierung der

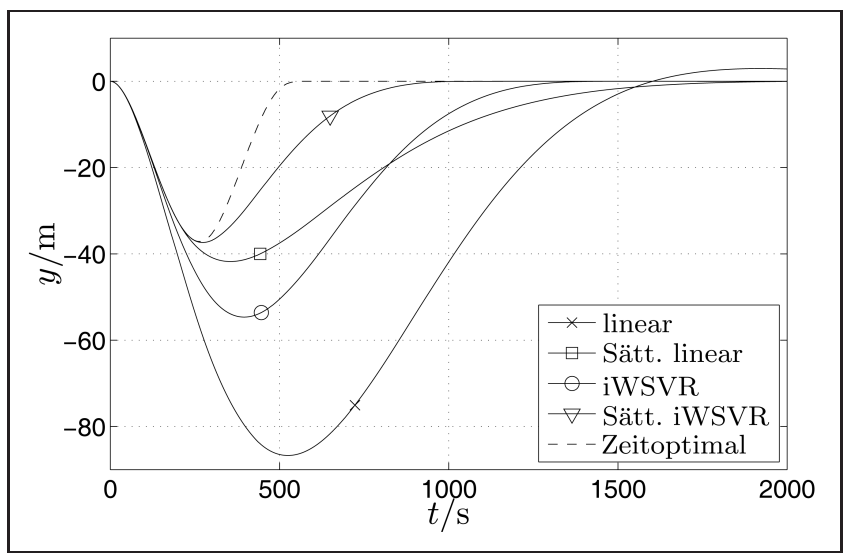

Bild 2: Tauchtiefe $y$ - LMI-basierter Entwurf.

Parameter mittels einer Evolutionsstrategie. Als Startpopulation wurde das Ergebnis der LMI-Optimierung sowie Mutationen dieses Ergebnisses verwendet. So ist sichergestellt, dass die Startpopulation auf jeden Fall mindestens einen gültigen Parametersatz enthält.

Ziel der Optimierung ist eine Regelung mit einer möglichst kleinen Ausregelzeit $T_{\mathrm{a}}$ bezogen auf ein vorher gewähltes Toleranzband für beliebige Anfangszustände. Die Ausregelzeit ist jedoch keine stetige Funktion der Reglerparameter. Dies ist für die Optimierung ungünstig, weshalb stattdessen das Gütemaß

$$
\sum_{i} \frac{1}{T^{4}} \int_{0}^{T}\left|y_{i}(t)\right| \cdot t^{4} \mathrm{~d} t,
$$

für die evolutionäre Optimierung verwendet wurde, wobei $T$ vor der Optimierung ausreichend groß gewählt wurde. Im Gütemaß (65) bezeichnet $y_{i}(t)$ den Ausgangsverlauf für den Anfangswert $x_{0, i}$. Die Anfangswerte $x_{0, i}$ durchlaufen alle Eckpunkte von $\mathcal{X}_{0}$. So wird sichergestellt, dass die Regelung nicht nur auf einen einzigen Anfangszustand optimiert wird.

Der Ausgang geht in das Gütemaß nicht, wie sonst oft üblich, quadratisch, sondern als Betrag ein. Es könnte sonst der Fall auftreten, dass große Auslenkungen, die durch eine

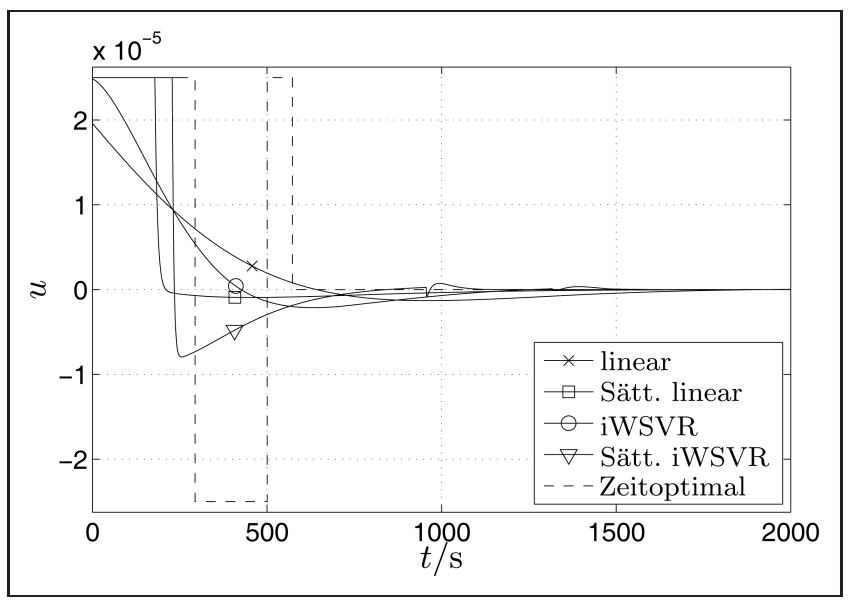

Bild 3: Stellgröße $u$ - LMI-basierter Entwurf. 


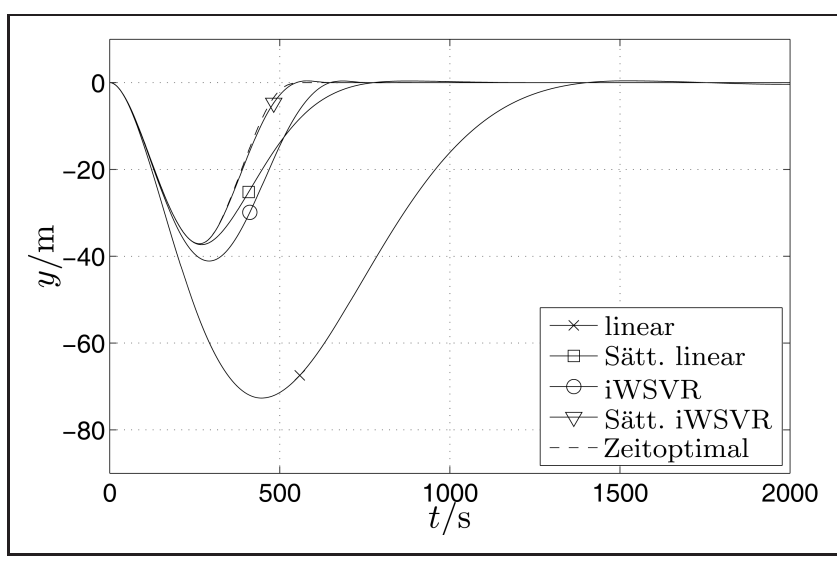

Bild 4: Tauchtiefe $y$ - Entwurf mittels Evolutionsstrategie.

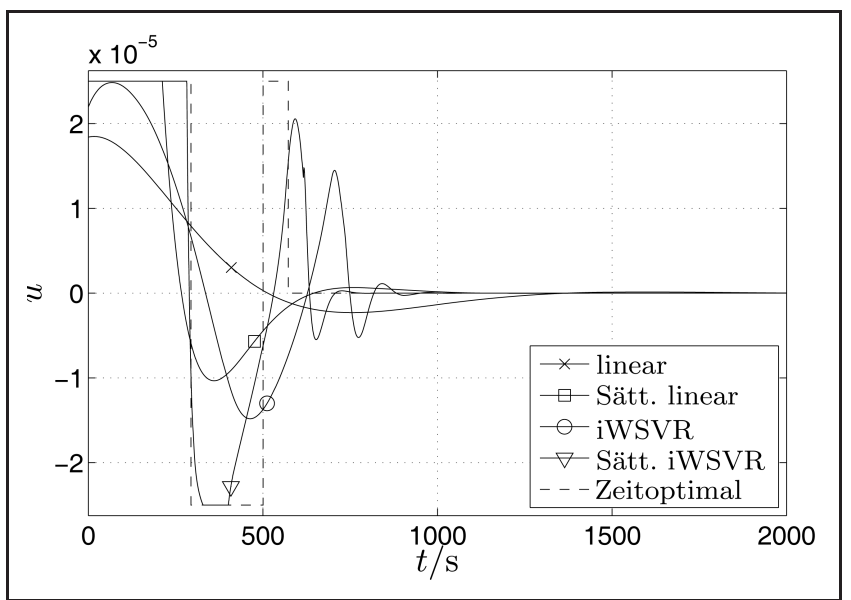

Bild 5: Stellgröße $u$ - Entwurf mittels Evolutionsstrategie.

Quadrierung stärker bestraft würden, auf Kosten einer längeren Ausregelzeit vermieden werden. Das Ziel ist jedoch eine kurze Ausregelzeit unabhängig von der maximalen Auslenkung von $y$. Durch die starke zeitliche Gewichtung mit dem Term $t^{4}$ in Gl. (65) werden Regler mit sehr schnellen Ausregelzeiten ermittelt.

Eine evolutionäre Optimierung liefert zwar nicht garantiert das globale Optimum, aber wie Bild 4 und die Vergröße-

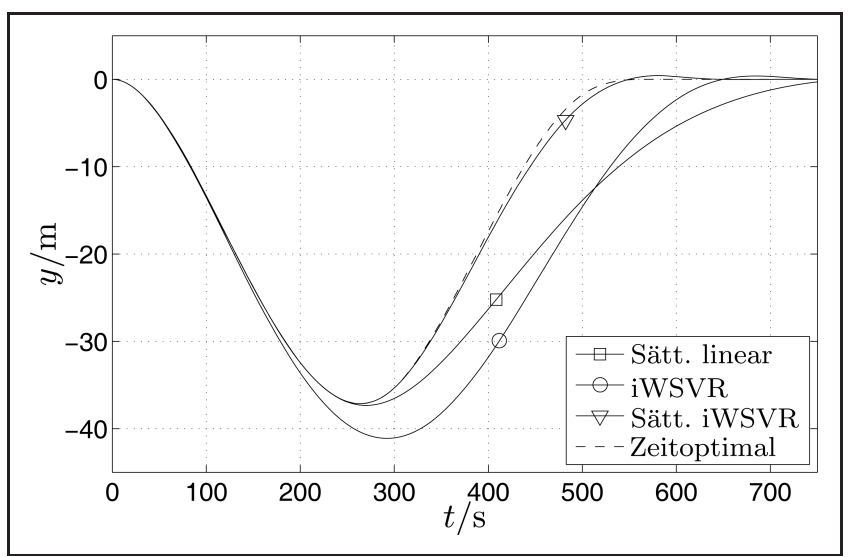

Bild 6: Vergrößerung von Bild 4.

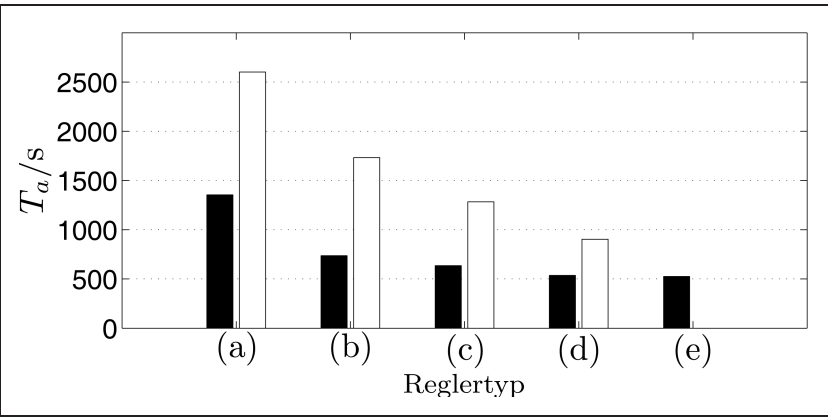

Bild 7: Vergleich der Ausregelzeiten $T_{\mathrm{a}}$ für das U-Boot (Toleranzband $\pm 0,5 \mathrm{~m})$. Weiß: Entwurf mittels konvexer Optimierung, Schwarz: Entwurf mittels Evolutionsstrategie, (a) linearer Regler, (b) sättigender linearer Regler, (c) implizite WSVR, (d) sättigende implizite WSVR, (e) zeitoptimal.

rung in Bild 6 zeigen, ist die sättigende implizite WSVR noch einmal deutlich schneller als in Bild 2 und äußerst nah am zeitoptimalen Verlauf. In Bild 5, in dem die Stellgrößenverläufe gezeigt werden, ist außerdem zu erkennen, dass der Verlauf der sättigenden impliziten WSVR dem zeitoptimalen Verlauf ähnelt, jedoch keine Unstetigkeiten in der Stellgröße aufweist. Diesen Performancegewinn erkauft man sich allerdings durch lange Rechenzeiten beim Entwurf, die abhängig von der Systemordnung mehrere Stunden betragen können.

Bild 7 stellt abschließend die Ausregelzeiten bezogen auf das Toleranzband $\pm 0,5 \mathrm{~m}$ dar. Man stellt fest, dass die sättigende WSVR unabhängig von der Entwurfsmethode das beste Ergebnis liefert. Auch wenn erwartungsgemäß alle Regler von der evolutionären Optimierung profitieren, bleibt die Reihenfolge gleich.

\section{Zusammenfassung}

In diesem Beitrag wurde gezeigt, wie sich sättigende Regler und implizite weiche strukturvariable Regler zu einem neuen Regler kombinieren lassen. Dieser sättigende implizite WSVR kann sehr effizient mittels konvexer Optimierungsmethoden entworfen werden. Für das oben gezeigte Beispiel ist die Regelung sehr schnell, bei anschließender nichtkonvexer Optimierung, beispielsweise durch Evolutionsstrategien, wird sogar fast zeitoptimale Performance erreicht. Eine vergleichbare Regelgüte konnte mit der sättigenden impliziten WSVR auch bei anderen Regelstrecken erzielt werden.

\section{Anhang}

Für die sättigenden Regler wurde $\beta=50$, für die WSVR $p_{\min }=0,1$ gewählt. Die konvexe Optimierung wurde mit dem Solver SDPT ${ }^{3}$ [17] zusammen mit dem Paket YALMIP [15] durchgeführt. Sie lieferte bei Verwendung des Optimierungsproblems (62) folgende Ergebnisse²:

\footnotetext{
${ }^{2}$ Die Angaben wurden auf vier signifikante Stellen gerundet. Aus Platzgründen wird die Ljapunov-Matrix $\mathbf{R}$ nur dann angegeben, wenn sie für das Regelgesetz benötigt wird.
} 


\begin{tabular}{|c|c|}
\hline Lineare $\mathrm{R}$. & $\mathbf{k}^{\mathrm{T}}=\left[5,989 \cdot 10^{-8} 3,648 \cdot 10^{-5} 0,0049\right]$ \\
\hline $\begin{array}{l}\text { Sättigende } \\
\text { lineare R. }\end{array}$ & $\begin{array}{l}\mathbf{h}^{\mathrm{T}}=\left[\begin{array}{lll}1,030 \cdot 10^{-7} & 4,043 \cdot 10^{-5} & 0,004261\end{array}\right] \\
\mathbf{k}^{\mathrm{T}}=\left[\begin{array}{lll}1,857 \cdot 10^{-7} & 9,308 \cdot 10^{-4} & 0,1417\end{array}\right]\end{array}$ \\
\hline Implizite WSVR & $\begin{array}{l}\hat{\mathbf{a}}^{\mathrm{T}}=\left[\begin{array}{lll}3,623 \cdot 10^{-8} & 3,162 \cdot 10^{-5} & 0,009809\end{array}\right] \\
\mathbf{R}_{1}=\left[\begin{array}{ccc}4,197 \cdot 10^{-6} & 0,002245 & 0,2782 \\
0,002245 & 1,681 & 243,1 \\
0,2782 & 243,1 & 40439\end{array}\right]\end{array}$ \\
\hline $\begin{array}{l}\text { Sättigende } \\
\text { implizite WSVR }\end{array}$ & $\begin{array}{l}\hat{\mathbf{a}}^{\mathrm{T}}=\left[\begin{array}{lll}7,01 \cdot 10^{-8} & 3,775 \cdot 10^{-5} & 0,009718\end{array}\right] \\
\hat{\mathbf{a}}^{\star \mathrm{T}}=\left[\begin{array}{lll}2,009 \cdot 10^{-6} & 0,001081 & 0,1486\end{array}\right] \\
\mathbf{R}_{1}=\left[\begin{array}{ccc}1,573 \cdot 10^{-5} & 0,005791 & 0,5298 \\
0,005791 & 2,588 & 285,1 \\
0,5298 & 285,1 & 38104\end{array}\right]\end{array}$ \\
\hline
\end{tabular}

Die Optimierung mittels einer Evolutionsstrategie mit dem Gütemaß (65) ergab die folgenden Werte:

\begin{tabular}{|c|c|}
\hline Lineare R. & 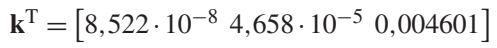 \\
\hline $\begin{array}{l}\text { Sättigende } \\
\text { lineare R. }\end{array}$ & $\begin{aligned} \mathbf{h}^{\mathrm{T}} & =\left[\begin{array}{lll}1,453 \cdot 10^{-7} & 4,491 \cdot 10^{-5} & 0,00414\end{array}\right] \\
\mathbf{k}^{\mathrm{T}} & =\left[\begin{array}{lll}9,958 \cdot 10^{-7} & 0,0002502 & 0,02018\end{array}\right]\end{aligned}$ \\
\hline Implizite WSVR & 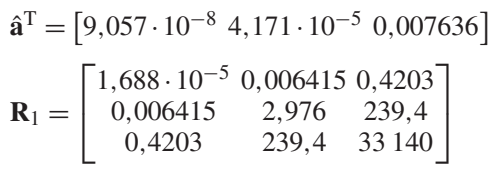 \\
\hline $\begin{array}{l}\text { Sättigende } \\
\text { implizite WSVR }\end{array}$ & $\begin{array}{l}\hat{\mathbf{a}}^{\mathrm{T}}=\left[\begin{array}{lll}8,792 \cdot 10^{-8} & 3,353 \cdot 10^{-5} & 0,008188\end{array}\right] \\
\hat{\mathbf{a}}^{\star \mathrm{T}}=\left[\begin{array}{lll}1,369 \cdot 10^{-6} & 0,000356 & 0,04775\end{array}\right] \\
\mathbf{R}_{1}=\left[\begin{array}{ccc}3,127 \cdot 10^{-5} & 0,00699 & 0,7973 \\
0,00699 & 2,098 & 237,1 \\
0,7973 & 237,1 & 36820\end{array}\right]\end{array}$ \\
\hline
\end{tabular}

\section{Literatur}

[1] Adamy, J.: Strukturvariable Regelungen mittels impliziter Ljapunov-Funktionen. VDI Verlag, 1991 (FortschrittBerichte VDI, Reihe 8).

[2] AdAmy, J.: Implicit Lyapunov Functions and Isochrones of Linear Systems. In: IEEE Transactions on Automatic Control 50 (2005), Nr. 6, S. 874-879.

[3] Adamy, J.; Flemming, A.: Soft variable-structure controls: a survey. In: Automatica 40 (2004), Nr. 11, S. 18211844.

[4] Adamy, J.; Lens, H.: Stabilitätsnachweis für weiche strukturvariable Regelungen mit Zustandsbeobachter. In: at Automatisierungstechnik 55 (2007), Nr. 3, S. 107-118.

[5] Boyd, S.; Ghaoui, L. E.; Feron, E.; Balakrishnan, V.: Linear Matrix Inequalities in System and Control Theory. Philadelphia: SIAM, 1994.

[6] Buhl, M.; Joos, P.; Lohmann, B.: Sättigende weiche strukturvariable Regelung. In: at - Automatisierungstechnik 56 (2008), Nr. 6, S. 316-323.

[7] CAO, Y.; Lin, Z.; Shamash, Y.: Set invariance analysis and gain-scheduling control for LPV systems subject to actuator saturation. In: Systems \& Control Letters 46 (2002), S. 137151.

Verfügbar unter

lediglich die vom Gesetz vorgesehenen Nutzungsrechte gemäß UrhG
[8] Chen, B. M.; Lee, T. H.; Peng, K.; VenkataramaNAN, V.: Composite nonlinear feedback control for linear systems with input saturation: theory and an application. In: IEEE Transactions on Automatic Control 48 (2003), Nr. 3, S. 427-439.

[9] De DonÁ, J. A.; Goodwin, G. C.; Moheimani, S. O. R.: Combining switching, over-saturation and scaling to optimise control performance in the presence of model uncertainty and input saturation. In: Automatica 38 (2002), Nr. 7, S. 1153-1162.

[10] Gutman, P.-O.; Hagander, P.: A new design of constrained controllers for linear systems. In: IEEE Transactions on Automatic Control 30 (1985), Nr. 1, S. 22-33.

[11] Hu, T.; Lin, Z.: Control Systems with Actuator Saturation. Birkhäuser, 2001.

[12] Hu, T.; Lin, Z.: On Improving the Performance With Bounded Continuous Feedback Laws. In: IEEE Transactions on Automatic Control 47 (2002), S. 1570-1575.

[13] Larsson, P.; Ulsoy, A.: Fast control of linear systems subject to input constraints. In: Transactions of the ASME 122 (2000), S. 18-26.

[14] Lin, Z.; PAChter, M.; BAndA, S.: Toward improvement of tracking performance - nonlinear feedback for linear systems. In: International Journal of Control 70 (1998), S. 1-11.

[15] Löfberg, J.: YALMIP: A Toolbox for Modeling and Optimization in Matlab. In: Proceedings of the CACSD Conference. Taipei, Taiwan, 2004.

[16] Rouche, N.; Habets, P.; Laloy, M.: Stability theory by Liapunov's direct method. New York: Springer-Verlag, 1977.

[17] Toh, K.-C.; Todd, M. J.; Tutuncu, R. H.: $\quad S D P T^{3} \quad$ a MATla software for semidefinite-quadratic-linear programming. http: / / www. math. nus.edu.sg/ mattohkc/ sdpt3.html. Version: 2006. -4.0 beta.

[18] Wu, S.-T.: Time-optimal control and high-gain linear static feedback. In: International Journal of Control 72 (1999), Nr. 9, S. 764-772.

[19] Yankulova, D.; A DAMY, J.: LMI-Entwurf schneller Regler mit impliziten Ljapunov-Funktionen. In: at - Automatisierungstechnik 56 (2008), Nr. 12, S. 636-643.

Manuskripteingang: 25. August 2008.

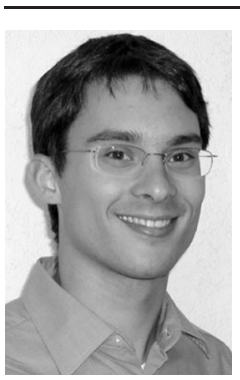

Dipl.-Ing. Hendrik Lens promoviert am Fachgebiet Regelungstheorie und Robotik der TU Darmstadt im Bereich nichtlineare Regelungen. Hauptarbeitsgebiete: weiche strukturvariable Regelungen, Systeme mit Stellgrößenbeschränkungen.

Adresse: TU Darmstadt, Fachbereich Elektro- und Informationstechnik, Fachgebiet Regelungstheorie und Robotik, Landgraf-Georg-Str. 4, 64283 Darmstadt, E-Mail: hlens@ @rtr.tu-darmstadt.de

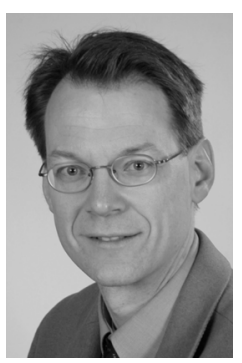

Prof. Dr.-Ing. Jürgen Adamy ist Leiter des Fachgebietes Regelungstheorie und Robotik im Fachbereich Elektro- und Informationstechnik der TU Darmstadt. Hauptarbeitsgebiete: Regelungsverfahren, Computational Intelligence, autonome mobile Roboter.

Adresse: TU Darmstadt, Fachbereich Elektro- und Informationstechnik, Fachgebiet Regelungstheorie und Robotik, Landgraf-Georg-Str. 4, 64283 Darmstadt, Fax: +49-(0)6151 162507 ,

E-Mail: adamy@ @rtr.tu-darmstadt.de 